\title{
A MULTIVARIATE ANALYSIS OF CARDIOPULMONARY PARAMETERS IN ARCHERY PERFORMANCE
}

original paper

( ) University School of Physical Education in Wroclaw

DOI: https://doi.org/10.5114/hm.2018.77322

\section{VIJAYAMURUGAN ESWARAMOORTHI ${ }^{1}$, MOHAMAD RAZALI ABDULLAH ${ }^{2}$, RABIU MUAZU MUSA ${ }^{2}$, AHMAD BISYRI HUSIN MUSAWI MALIKI' ${ }^{2}$, NORLAILA AZURA KOSNI ${ }^{2}$, NARESH BHASKAR RAJ ${ }^{1}$, NORZULAIKA ALIAS ${ }^{1}$, HANISAH AZAHARI ${ }^{1}$, SITI MUSLIHA MAT-RASHID ${ }^{2}$, HAFIZAN JUAHIR ${ }^{3}$}

\author{
${ }^{1}$ Faculty of Health Sciences, School of Rehabilitation Science, University of Sultan Zainal Abidin, Terengganu, Malaysia \\ ${ }^{2}$ Faculty of Applied Social Sciences, University of Sultan Zainal Abidin, Terengganu, Malaysia \\ ${ }^{3}$ East Coast Environmental Research Institute, University of Sultan Zainal Abidin, Terengganu, Malaysia
}

\begin{abstract}
Purpose. The aim of this investigation was to determine the most significant cardiopulmonary parameters bound with high archery scores and to assess their relationship with successful performance in archery.

Methods. The total of 32 archers with mean age of $17 \pm 0.56$ years were gathered from dissimilar archery programmes. Cardiopulmonary parameters were measured prior to shooting tests. Multivariate techniques of principal component analysis (PCA), hierarchical agglomerative cluster analysis (HACA), and discriminant analysis (DA) were used to analyse the data collected.

Results. The initial PCA identified 4 parameters with a higher eigenvalue ( $>1)$. However, PCA after varimax rotation indicated 4 varifactors with high positive loadings, containing 3 respiratory parameters: forced vital capacity (FVC; 0.83), maximum voluntary ventilation (MVV; 0.83), and peak expiratory flow rate (PEFR; 0.87); 2 pressure parameters: resting diastolic blood pressure (RDBP; 0.78 ) and resting systolic blood pressure (RSBP; 0.88); 1 volume parameter: inspiratory reserve volume (IRV; 0.86); and 1 rate parameter: resting respiratory rate (RRR; 0.87). HACA divided the archers into 2 categories on the basis of their performance on the most needed parameters; these were high-optimum pulmonary capacity archers (HOCA) and low-optimum pulmonary capacity archers (LOCA). Standard, backward stepwise, and forward stepwise DA discriminated the classes from the 7 parameters with remarkable accuracy $(90.63 \%, 93.75 \%$, and $96.88 \%$, respectively) for each method, confirming the classification provided by HACA.
\end{abstract}

Conclusions. It is obvious from the current outcomes that such cardiopulmonary parameters as good FVC, MVV, PEFR, IRV, RRR, and optimal RSBP and RDBP are necessary for better archery performance.

Key words: archery, cardiopulmonary, multivariate analysis, archery performance

\section{Introduction}

Archery is a fine and highly skilled Olympic sport; high performance in this discipline is characterized as the ability of shooting at the target repeatedly in a set amount of time with high precision and accuracy [1]. This involves conscious breath control and synchronization of breathing with limb movements [2]. In older times, archery was bound with hunting and also considered as a very formidable weapon of war. So, archery has been always perceived as a preferred skill as well as interesting hobby.

Shooting in archery involves concurrent matching between breathing; gross motor control of body positioning; fine-motor control of the archers' fingers, hands, elbows, legs, feet, and cheek; and the handling of perceptual cues associated with the target, the sights, and the peep or string [3]. Good archers need not only spe-

Correspondence address: Mohamad Razali Abdullah, Faculty of Applied Social Sciences, University of Sultan Zainal Abidin, Gong Badak Campus, Kuala Nerus, 21300, Terengganu, Malaysia, e-mail: razali896@yahoo.com

Received: December 17, 2017

Accepted for publication: February 21, 2018

Citation: Eswaramoorthi V, Abdullah MR, Musa RM, Maliki ABHM, Kosni NA, Raj NB, Alias N, Azahari H, Mat-Rashid SM, Juahir H. A Multivariate analysis of cardiopulmonary parameters in archery performance. Hum Mov. 2018;19(4):35-41; doi: https://doi.org/10.5114/hm.2018.77322. 
cific mental and physical characteristics but also precise skill and perception, as a minute shift in aiming leads to a serious problem [4].

Taking into account the essential role of accuracy in archery, Bartlett and Leiter [2] found that the breathing pattern of archers influenced the act of archery. Some other studies also prove that the breathing cycle and cardiac cycle potentially affect the aiming stability in archery [5]. Hung et al. [6] stressed the importance of breathing training in the successful performance of an Olympic archer.

Thakare [7] explored the peak expiratory flow rate (PEFR) in Indian archers and found high PEFR values among all studied archers compared with normal men. Studies strongly support the idea that cardiopulmonary parameters influence the performance in archery. The numerous cardiac parameters also include: resting heart rate (RHR), resting systolic blood pressure (RSBP), resting diastolic blood pressure (RDBP); the pulmonary parameters are: resting respiratory rate (RRR), tidal volume (Vt), inspiratory reserve volume (IRV), expiratory reserve volume (ERV), inspiratory capacity (IC), forced vital capacity (FVC), PEFR, maximum voluntary ventilation (MVV). Out of these parameters, it is still not clear which ones influence the game of archery most.

There is very limited research on these parameters and their relationship with archery. So, quantifying those which influence the performance in archery will hugely help to assist the sport to enhance the performance of its athletes. Therefore, this study examines the relationship between the cardiopulmonary parameters and the successful shooting in archery in order to find out the best significant variable that promotes reaching high archery scores.

\section{Material and methods}

\section{Participants}

With ex post facto design, the total of 32 non-smoking archers with no musculoskeletal, cardio-respiratory, or neurological disorders were enrolled to take part in the study. Among these, 24 were male and 8 were female youth archers; their age range was 13-24 years, with the mean and standard deviation of $17.0 \pm$ 0.56 . The archers came from the Terengganu sports council, Malaysia. The coaches and the participants were educated about the purpose of the research.

\section{Measurements}

Cardiac parameters such as RHR, RSBP, and RDBP of all 32 archers were measured with the use of an Om- ron automatic blood pressure monitor (HEM-7120), which is a reliable tool for measuring these parameters [8]. All specified cardiac parameters were measured while the archers were at rest and sitting comfortable on a chair. The device was placed at the chest level and the arm strap was attached securely on the left arm of the archers; then the strap was inflated automatically by the device, and the blood pressure and pulse rate measurements were noted.

The pulmonary parameter of Vt was measured with the slow vital capacity test. Vital capacity (VC), IRV, ERV, IC, FVC, PEFR, and MVV were determined with a digital Pony FX micro spirometer (Cosmed, Rome) [9]. All the measurements of the given pulmonary parameters were performed while the archers were sitting on a chair with their extremities and shoulders straight, legs spread shoulder-width apart and at right angles to the ground. The participants were then taught to close their nose via a nose clamp, grasp the assessing tool with one hand and let it nip the mouth [9].

FVC and PEFR measurements were taken when the archers took breath usually 3 or 4 times and then took a deep and fast inhalation tailed by a fast vigorous exhalation. The exhalation was sustained for 6 seconds. Slow vital capacity was then determined with the archers in a similar position; they were instructed to breathe at rest for some time, and then to perform a maximal inspiration following a slow forced exhalation.

MVV was then measured with the archers in an identical position. The participants were asked to accomplish inspiration and expiration for 12 seconds in the deepest and fastest fashion. Each parameter measurement was taken 3 times, and the average value of each was used in the analysis. RRR was measured in the same position; a therapist palpated the respiratory movements of the archers' chest for a minute, using a stop watch. All measurements were completed prior to the shooting score test.

For the archery shooting score test, an imitation shooting competition zone was arranged. All the archers shot 6 arrows (one end) over a distance of 50 meters. They performed 4 trial shots before the ultimate noted 6 arrow scores.

\section{Statistical analysis}

In the current study, normality was verified by means of the Shapiro-Wilk test and the archers were found to be homogeneously distributed. The principal component analysis (PCA) was employed with the intention of giving insights into the most important parameter due to the dissimilarities of relative perfor- 
mance variables that describe the entire data set, by reducing numerous variables with a significantly limited loss of the original data [10]. Hierarchical agglomerative cluster analysis (HACA) was used to segregate the classes of the relevant performance variables measured. Discriminant analysis (DA) was applied with the use of the standard, forward stepwise, and backward stepwise methods. These techniques were employed to build degrees of freedom to evaluate relative performance variations in cardiopulmonary parameters, as well as the archery shooting performance. The relative performances of the archers were the gathered (dependent) variables while all the evaluated parameters constituted the independent variables. Likewise, in the forward stepwise mode, the variables were calculated step by step, starting with the most significant variable until no significant changes were obtained. In the backward stepwise mode, variables were removed step by step, opening with the least essential variable until no significant changes were attained. All the statistical investigation was performed by means of GraphPad Prism version 4.03 and XLSTAT version 2014 addin software (New York, USA) with the significance level of $\alpha=0.05$.

\section{Ethical approval}

The research related to human use has been complied with all the relevant national regulations and institutional policies, has followed the tenets of the Declaration of Helsinki. All the procedures, rules, and devices for the study were approved by the Research Ethics Board of the Terengganu Sports Institute (ISNT), reference number 04-04/T-01/Jid 2.

\section{Informed consent}

Informed consent has been obtained from all individuals included in this study.

\section{Results}

Table 1 demonstrates the descriptive statistics of the variables evaluated. The number of the participants, the minimum and maximum scores, mean, as well as standard deviation of each parameter is shown.

Table 2 illustrates the Kaiser-Meyer-Olkin (KMO) measure of sampling adequacy. This investigation was useful to verify the adequacy of the sampling to calculate, as well as to sort a rational analysis constructed on the data collected. Likewise, the investigation was performed to confirm that the variables were not associated with each other. The KMO value in Table 2 equals 0.53 , which points out that the numbers of the
Table 1. Descriptive statistics of the evaluated variables

\begin{tabular}{lcrrrr}
\hline Variable & $n$ & Min. & \multicolumn{1}{c}{ Max. } & \multicolumn{1}{c}{$M$} & \multicolumn{1}{c}{$S D$} \\
\hline RHR (beats/min) & 32 & 61.00 & 101.00 & 84.00 & 10.97 \\
RRR (breaths/min) & 32 & 11.00 & 30.00 & 19.56 & 5.02 \\
RSBP (mm Hg) & 32 & 95.00 & 144.00 & 118.81 & 12.97 \\
RDBP (mm Hg) & 32 & 57.00 & 94.00 & 75.13 & 8.66 \\
FVC (ml/kg) & 32 & 2.07 & 5.19 & 3.79 & 0.79 \\
MVV (l/s) & 32 & 47.50 & 150.90 & 96.32 & 24.93 \\
PEFR (l/s) & 32 & 3.70 & 11.07 & 7.41 & 1.69 \\
ERV (ml/kg) & 32 & 0.01 & 6.54 & 1.05 & 1.23 \\
IC (l/s) & 32 & 1.48 & 7.89 & 3.56 & 1.16 \\
VC (ml/kg) & 32 & 1.73 & 5.23 & 3.19 & 0.83 \\
IRV (ml/kg) & 32 & 0.33 & 5.49 & 2.15 & 1.20 \\
Vt (ml/kg) & 32 & 0.16 & 2.60 & 1.41 & 0.71 \\
\hline RHR - restng
\end{tabular}

RHR - resting heart rate, $R R R$ - resting respiratory rate, RSBP - resting systolic blood pressure, RDBP - resting diastolic blood pressure, FVC - forced vital capacity, MVV - maximum voluntary ventilation, PEFR - peak expiratory flow rate, ERV - expiratory reserve volume, IC - inspiratory capacity, VC - vital capacity, IRV - inspiratory reserve volume, $\mathrm{Vt}$ - tidal volume

Table 2. Kaiser-Meyer-Olkin (KMO) measure of sampling adequacy

\begin{tabular}{ll}
\hline Variable & Value \\
\hline RHR (beats/min) & 0.64 \\
RRR (breaths/min) & 0.54 \\
RSBP (mm Hg) & 0.53 \\
RDBP (mm Hg) & 0.33 \\
FVC (ml/kg) & 0.86 \\
MVV (l/s) & 0.52 \\
PEFR (l/s) & 0.78 \\
ERV (ml/kg) & 0.28 \\
IC $(\mathrm{l} / \mathrm{s})$ & 0.47 \\
VC (ml/kg) & 0.83 \\
IRV (ml/kg) & 0.37 \\
Vt $(\mathrm{ml} / \mathrm{kg})$ & 0.27 \\
KMO & 0.53 \\
\hline
\end{tabular}

RHR - resting heart rate, RRR - resting respiratory rate, RSBP - resting systolic blood pressure, RDBP - resting diastolic blood pressure, FVC - forced vital capacity, MVV - maximum voluntary ventilation, PEFR - peak expiratory flow rate, ERV - expiratory reserve volume, IC - inspiratory capacity, VC - vital capacity, IRV - inspiratory reserve volume, $\mathrm{Vt}$ - tidal volume

contributors are satisfactory to make a realistic interpretation. Hence, on the basis of these outcomes, it is apparent that there is no multicollinearity identified amongst the original variables; this allowed us to continue with the PCA analysis, having fulfilled the measure of the sampling adequacy. 

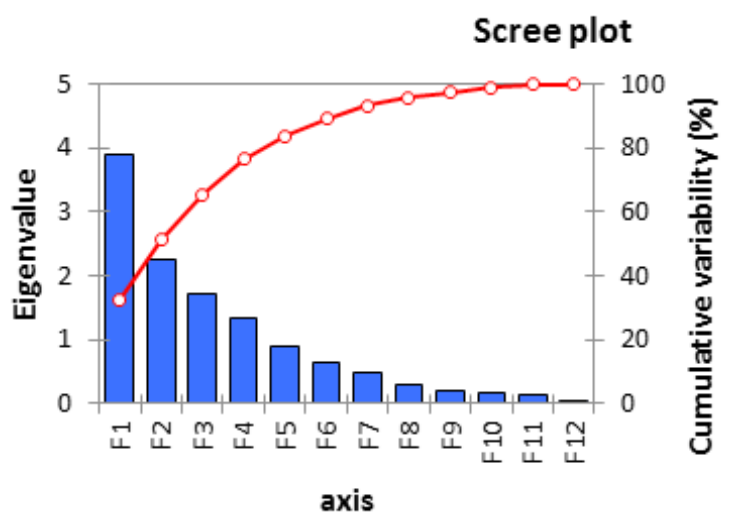

Figure 1. Scree plot for the principal component analysis

Table 3. Principal component analysis factor loading after varimax rotation

\begin{tabular}{lrrrr}
\hline Variables & VF1 & VF2 & VF3 & \multicolumn{1}{c}{ VF4 } \\
\hline RHR (beats/min) & -0.041 & 0.237 & -0.257 & 0.669 \\
RRR (breaths/min) & -0.031 & 0.030 & 0.102 & $0.876^{*}$ \\
RSBP (mm Hg) & 0.216 & $0.785^{*}$ & 0.072 & 0.184 \\
RDBP (mm Hg) & -0.207 & $0.880^{*}$ & 0.093 & 0.142 \\
FVC (ml/kg) & $0.839^{*}$ & 0.118 & 0.283 & -0.232 \\
MVV (1/s) & $0.838^{*}$ & -0.052 & -0.301 & 0.205 \\
PEFR (l/s) & $0.879^{*}$ & 0.025 & 0.245 & -0.008 \\
ERV (ml/kg) & 0.075 & -0.010 & 0.623 & 0.247 \\
IC (l/s) & 0.323 & 0.316 & 0.682 & -0.449 \\
VC (ml/kg) & 0.660 & 0.342 & 0.438 & -0.300 \\
IRV (ml/kg) & 0.074 & -0.067 & $0.864^{*}$ & -0.295 \\
Vt (ml/kg) & 0.404 & 0.631 & -0.347 & -0.236 \\
Eigenvalue & 3.906 & 2.236 & 1.698 & 1.323 \\
Variability (\%) & 32.552 & 18.634 & 14.148 & 11.026 \\
Cumulative (\%) & 32.552 & 51.185 & 65.334 & 76.360 \\
\hline
\end{tabular}

* Values that met the factor loading threshold of 0.75

$\mathrm{VF}$ - varimax factor, RHR - resting heart rate, RRR - resting respiratory rate, $\mathrm{RSBP}$ - resting systolic blood pressure, RDBP - resting diastolic blood pressure, FVC - forced vital capacity, MVV - maximum voluntary ventilation, PEFR - peak expiratory flow rate, ERV - expiratory reserve volume, IC - inspiratory capacity, VC - vital capacity, IRV - inspiratory reserve volume, $\mathrm{Vt}$ - tidal volume

Figure 1 reveals the eigenvalue after the initial PCA, which shows that the PCA identified 4 components as the most significant owing to their higher eigenvalues (>1). These components were retained and used for further analysis.

Table 3 presents the PCA after varimax rotation. It can be observed that 3 parameters from VF1, 2 from VF2, and 1 from VF3 and VF4 each fulfilled the 0.75 factor loading threshold.

Figure 2 illustrates the most significant parameters after varimax rotation; the contribution of each varifactor within the components, as well as their variability

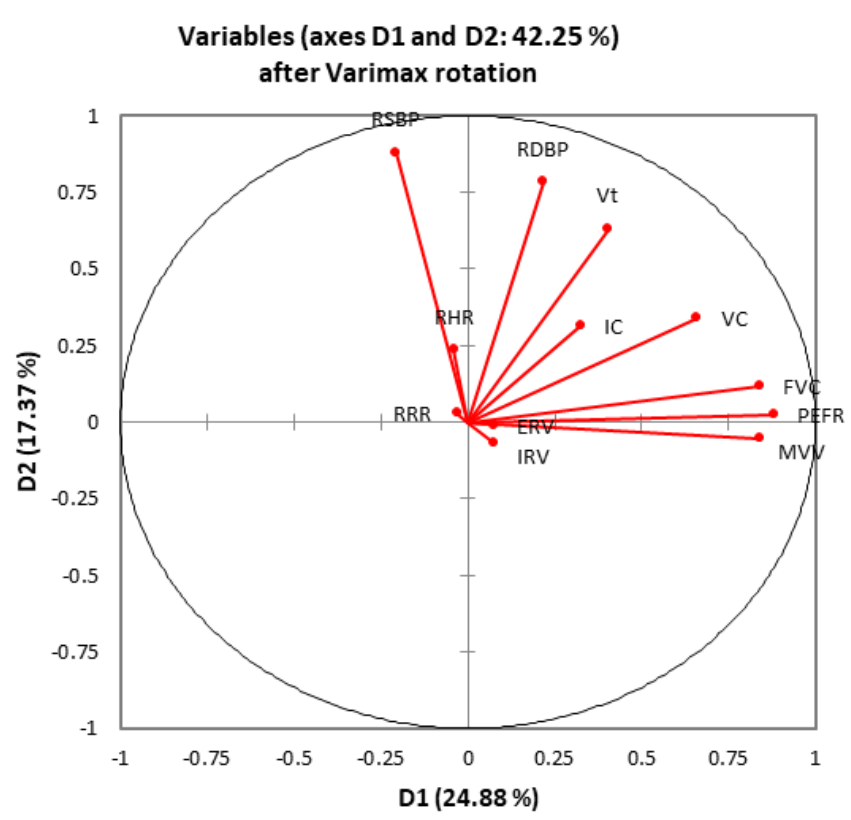

$\mathrm{RHR}$ - resting heart rate, RRR - resting respiratory rate, RSBP - resting systolic blood pressure, RDBP - resting diastolic blood pressure, FVC - forced vital capacity, MVV - maximum voluntary ventilation, PEFR - peak expiratory flow rate, ERV - expiratory reserve volume, IC - inspiratory capacity, VC - vital capacity, IRV - inspiratory reserve volume, $\mathrm{Vt}$ - tidal volume

Figure 2. Factor loading plot after varimax rotation

are also shown. It can be noticed that the varimax factors (VF) VF1, VF2, VF3, and VF4 contributed to about $42.25 \%$ of the total data set. Likewise, a variability of $24.88 \%$ and $17.37 \%$ is observed, which explains that the components differ with a variation of approximately $24 \%$ and $17 \%$, respectively.

Figure 3 displays the grouping of the archers in relation to their performance classes determined by HACA, which is based on the similarity level of the relative performance parameters evaluated. It can be observed that 2 classes are defined by HACA, namely: high-optimum pulmonary capacity (HOCA) archers and low-optimum pulmonary capacity (LOCA) archers. This is based on their scores on the evaluated parameters; the corresponding observations are given under each cluster.

Figure 4 presents group profile plot of each cluster of relative performance on the measured variables. The performance of the archers based on the variables examined is depicted. It can be concluded that the HOCA archers presented the highest performance across the 4 variables tested (FVC, MVV, PEFR, shooting scores). The LOCA archers obtained the lowest scores on the same 4 variables examined. This clustering facilitated the classification and assignation of the performance-related variables to the athletes and subsequently directed us to the further analysis to confirm the differentiation of archers. 


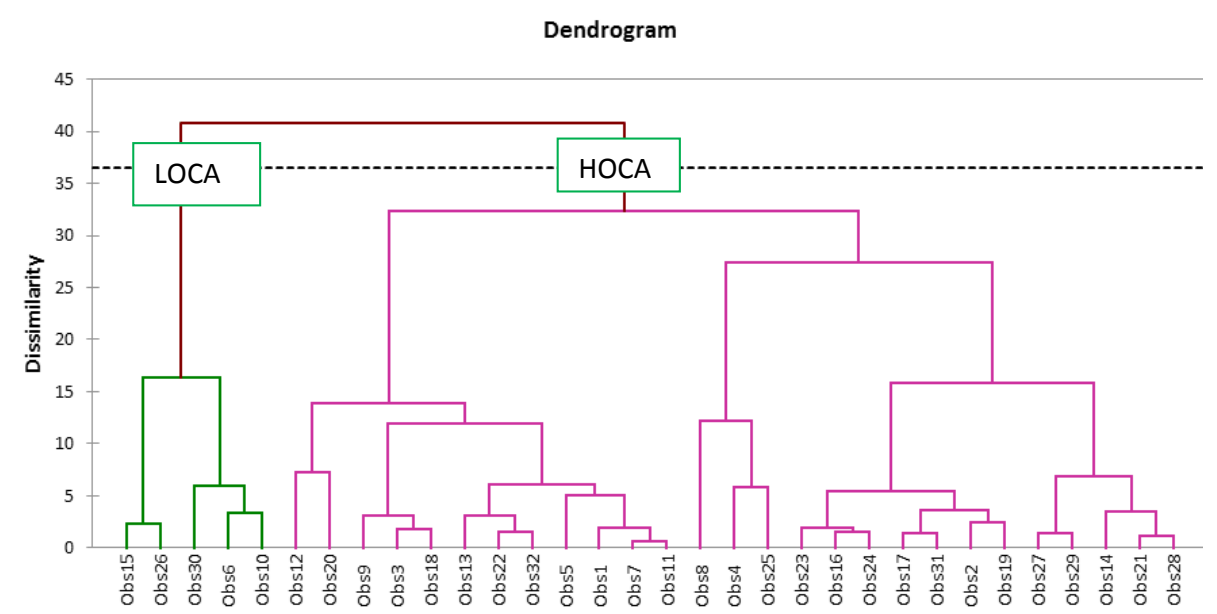

LOCA - low-optimum pulmonary capacity HOCA - high-optimum pulmonary capacity Obs - observation
Table 4. Classification matrix of the discriminant analysis (DA) of the 2 classes in relation to their performance on the variables measured

\begin{tabular}{lccc}
\hline & & \multicolumn{2}{c}{$\begin{array}{c}\text { Classification matrix } \\
\text { assigned by DA }\end{array}$} \\
\cline { 2 - 4 } & \multicolumn{2}{c}{ HOCA } & LOCA \\
\hline Standard mode (4 independent variables) & \\
\hline HOCA & $92.59 \%$ & 25 & 1 \\
LOCA & $80.00 \%$ & 2 & 4 \\
Total & $90.63 \%$ & 27 & 5 \\
\hline Backward stepwise & $(3$ independent variables $)$ & \\
\hline HOCA & $96.30 \%$ & 26 & 1 \\
LOCA & $80.00 \%$ & 1 & 4 \\
Total & $93.75 \%$ & 27 & 5 \\
\hline Forward stepwise $(1$ independent variable) & \\
\hline HOCA & $100.00 \%$ & 27 & 1 \\
LOCA & $80.00 \%$ & 0 & 4 \\
Total & $96.88 \%$ & 27 & 5 \\
\hline
\end{tabular}

HOCA - high-optimum pulmonary capacity,

LOCA - low-optimum pulmonary capacity

Table 4 illustrates the DA conducted for further analysis. The DA was applied to clusters defined by HACA in order to view through variation of relative performance. The clusters act as the independent variable, while the cardiopulmonary variables as well as the archery shooting scores were treated as dependent variables. Standard, backward stepwise, and forward stepwise methods were selected to perform the DA. The precision of classification with all the 3 methods were excellent $(90.63 \%, 93.75 \%$, and $96.88 \%$ with 4,3 , and 1 independent variable, respectively). Similarly, we found that 27 archers were classified to HOCA while 5 archers into LOCA.

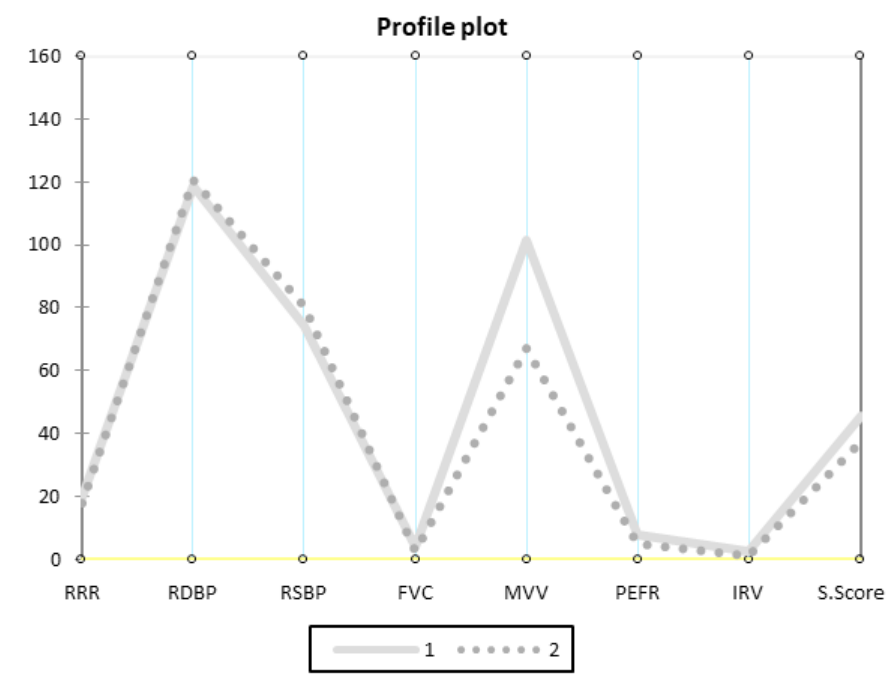

1 - high-optimum pulmonary capacity (HOCA), 2 - low-optimum pulmonary capacity (LOCA), RRR - resting respiratory rate, RDBP - resting diastolic blood pressure, RSBP - resting systolic blood pressure, FVC - forced vital capacity, MVV - maximum voluntary ventilation, PEFR - peak expiratory flow rate, IRV - inspiratory reserve volume, S.Score - shooting score

Figure 4. Profile plot of the 2 classes in relation to their performance on the measured variables

\section{Discussion}

The present study aimed to determine the connection between cardiopulmonary parameters and successful shooting in archery, as well as to find out the best significant parameter that contributes to reaching high archery scores. To attain the goals of the study, we recruited 32 archers from dissimilar archery programs in Terengganu, Malaysia. We measured the archers' cardiopulmonary parameters. Their shooting scores were noted after the cardiopulmonary parameters measurement. We employed PCA, HACA, as well as DA statistical analysis to establish clusters of the archers to ascertain the most vital cardiopulmonary parameters supporting high archery shooting scores and to recognize the differentiating features of the clusters. 
V. Eswaramoorthi et al., Multivariate analysis of cardiopulmonary parameters in archery

HACA specified clusters of the performance groups (Figure 3). However, the principal components with absolute values larger than 0.75 for PCA were standardised as the selection threshold because these values were noticeably solid and stable, which shows moderate to strong loadings on the extracted factors. This demonstrated that 7 cardiopulmonary parameters satisfied the 0.75 factor loading threshold (Table 3, Figure 2). These parameters were then categorized as the vital parameters promoting higher archery shooting scores, though each contained varifactors associated to it. DA discriminated clusters on the basis of performance in the predicted parameters (Table 4, Figure 4).

VF1 contributed to about $32.55 \%$ of the accumulation of the cardiopulmonary parameter data. It presented high positive factor loadings from 3 parameters: FVC (0.83), PEFR (0.87), and MVV (0.83). Considering the structure of these 3 parameters, they can be described as the capacity of lung function. This result reveals the importance of high capacity of lung function in the sport of archery. The observation is consistent with the study of Thakare [7], who investigated the specific pulmonary parameter of PEFR among archers participating in all India inter-university archery competitions and found out that the capacity of lung function was high in all archers compared with normal men and that archers having dissimilar bows had a different capacity of lung function.

The FVC parameter findings of this study are in line with the statement in the book by Eston and Reilly [11]. They maintain that repeated training of shoulder muscles helps to enhance the VC, and, with reference to archery, that the repeated lifting of a bow increases the strength of accessory inspiration muscles. It is obvious that to hold a bow constantly for a long time and reach high performance in the discipline, high capacity of lung function and shoulder muscular endurance are needed, which was also supported by the finding that the release pause of breathing (particularly exhalation) force majorly contributed to higher shooting precision [12]. These results recommend archers to enhance their capacity of lung function in order to perform better in the sport of archery.

VF2, as presented in Table 3, accounted for about $51.18 \%$ of the accumulation in cardiopulmonary parameters data. It exhibits high positive loadings from RSBP (0.88) and RDBP (0.78); the mean value of RSBP was $118.81( \pm 12.97)$ and that of RDBP equaled 75.13 $( \pm 8.66)$ (Table 1). These parameters can be interpreted as optimum resting blood pressure. This points at the vitality of optimum resting blood pressure in archery.
Blood pressure is majorly regulated by the autonomic nervous system; loss of balance between the sympathetic and parasympathetic stimulation leads to too many variations, which in turn results in an increased level of anxiety and thus negatively affects performance in archery. These findings are in line with the research by Lo et al. [13], who concluded that the optimum balance of parasympathetic and sympathetic activity was advantageous to archery performance. Clemente et al. [14] also found that experienced archers had good arousal control.

VF3 contributed to about $65.33 \%$ of the accumulation of the cardiopulmonary parameters data. It had high positive factor loadings from 1 parameter, namely IRV (0.86), which can also be described as the capacity of lungs. This observation again proves the importance of good lung capacity in the game, and is supported by Eston and Reilly [11], who affirm that repeated training of shoulder muscles helps to enhance the VC since VC is calculated as the sum of IRV + Vt + ERV, and, notably, archery involves repeated activity of shoulder muscles.

VF4 added to about $76.36 \%$ of the accumulation of the cardiopulmonary parameters data. It had high positive factor loadings from 1 parameter, RRR (0.87), and the mean RRR value was 19.56 ( \pm 5.06$)$ (Table 1$)$. This parameter can be described as optimum lung frequency. Studies indicate that postural sway affects the accuracy of archery, which in turn is influenced by the breathing pattern [2]. As the postural shift is remarkably controlled while breath holding [15], the respiratory frequency of novice, elite, and experienced golfers aged 19-41 years during the performance of golf putting was 25 cycles per minute. The present study revealed the mean RRR value of archers to be 19/minute, which may be due to good arousal control and autonomic balance of the archers at rest, apparently supporting optimum lung frequency during rest and leading to good breath control and postural stability during the game for a better performance enhancement.

HACA was performed to classify the archers on the basis of their similarity on relative performance on the selected parameters in the study. The analysis recognized 2 groups: HOCA and LOCA, which were well distinguished with lung capacity parameters. DA discriminated clusters based on the participants' performance in the predicted parameters; the precision of classification in DA was $90.63 \%$ with the standard mode with FVC, MVV, PEFR, and IRV parameters; 93.75\% with the backward stepwise mode with FVC, MVV, and IRV parameters; and $96.88 \%$ with the forward stepwise mode with the PEFR parameter. This reveals the importance of (FVC, MVV, PEFR, and IRV) 
high capacity of lung function in archery, remaining consistent with the previous research $[7,11,12]$. The current study proved that lung capacity parameters, such as FVC, MVV, PEFR, and IRV, influenced performance in archery. Thus archers have to pay attention to these parameters to enhance their performance.

\section{Conclusions}

The present investigation has studied the impact of cardiopulmonary parameters on the successful act of archery. It has been recognized that the combination of good lung capacity, adequate frequency, and optimal resting blood pressure is crucial for archery performance. This discovery has shed more light on the cardiopulmonary parameters that are essential for successful performance in archery. The result can be beneficial to coaches and sports managers, making them understand the cardiopulmonary parameters significant in archery performance and guiding them to plan appropriate training programs to fit the requirements of the sport in association with the highlighted cardiopulmonary characteristics.

\section{Acknowledgements}

The authors acknowledge the Research and Development Management Unit, University of Sultan Zainal Abidin, Kuala Terengganu, for providing the internal research grant for the study (UniSZA/2016/DPU/17), and also the Community Cluster of University of Sultan Zainal Abidin, Kuala Terengganu, for the financial support provided to purchase the digital Pony FX micro spirometer (Cosmed, Rome). The authors also express their gratitude to the Terengganu State Sports Council for their support for this study. Moreover, the authors are thankful to all team managers, coaches, as well as the athletes for their help and cooperation throughout the data collection process.

\section{Disclosure statement}

No author has any financial interest or received any financial benefit from this research.

\section{Conflict of interest}

The authors state no conflict of interest.

\section{Funding}

This study was supported by an internal research grant (UniSZA/2016/DPU/17) from the Research and Development Management Unit, University of Sultan Zainal Abidin, Kuala Terengganu.

\section{References}

1. Vrbik A, Bene R, Vrbik I. Heart rate values and levels of attention and relaxation in expert archers during shooting. Croat Sports Med J. 2015;30(1):21-29.

2. Bartlett D, Leiter JC. Coordination of breathing with nonrespiratory activities. Compr Physiol. 2012;2(2): 1387-1415; doi: 10.1002/cphy.c110004.

3. Chung GK, Delacruz GC, de Vries LF, Bewley WL, Baker EL. Mil Psychol. 2006;18(2):161-179; doi: 10.1207/ s15327876mp1802_5.

4. Lin KB, Hwang CK. Analysis of correlation between aiming adjustment trajectory and target. J Mech Med Biol. 2005;5(2):253-259; doi: 10.1142/S02195194050 01412.

5. Mason BR, Pelgrim PP. Body stability and performance in archery. Excel. 1986;3(2):17-20.

6. Hung TM, Tang WT, Shiang TY. A case study of integrated sport sciences for an Olympic archer. J Med Biol Eng. 2009;29(4):164-171.

7. Thakare V. Comparative study of peak expiratory flow rate of archery players participated in all India inter university archery competition. Int J Phys Educ Sports Health. 2015;2(2):331-332.

8. Abdullah MR, Eswaramoorthi V, Musa RM, Maliki AB, Kosni NA, Haque M. The effectiveness of aerobic exercises at difference intensities of managing blood pressure in essential hypertensive information technology officers. J Young Pharm. 2016;8(4):483-486; doi: 10.5530/jyp.2016.4.27.

9. Han D, Ha M. Effect of pelvic floor muscle exercises on pulmonary function. J Phys Ther Sci. 2015;27(10): 3233-3235; doi: 10.1589/jpts.27.3233.

10. Abdullah MR, Maliki AB, Musa RM, Kosni NA, Juahir H, Haque M. Multi-hierarchical pattern recognition of athlete's relative performance as a criterion for predicting potential athletes. J Young Pharm. 2016; 8(4):463-470; doi: 10.5530/jyp.2016.4.24.

11. Eston R, Reilly T (eds.). Kinanthropometry and exercise physiology laboratory manual. New York: Routledge; 2013.

12. Mohamed MN, Norman WM, Linoby A, Sariman MH, Azam MZ. Breathing pattern influence to the shooting performance. In: Adnan R, Ismail S, Sulaiman N (eds.), Proceedings of the International Colloquium on Sports Science, Exercise, Engineering and Technology 2014 (ICoSSEET 2014). Singapore: Springer; 2014; 321-333.

13. Lo CT, Huang SH, Hung TM. A study of the relationship between heart rate variability and archery performance. Int J Psychophysiol. 2008;69(3):276-316; doi: 10.1016/ j.ijpsycho.2008.05.231.

14. Clemente FM, Couceiro MS, Rocha R, Mendes R. Study of the heart rate and accuracy performance of archers. J Phys Educ Sport. 2011;11(4):434-437; doi: 10.1007/ s11274-015-1903-5.

15. Neumann DL, Thomas PR. The relationship between skill level and patterns in cardiac and respiratory activity during golf putting. Int J Psychophysiol. 2009;72(3):276282; doi: 10.1016/j.ijpsycho.2009.01.001. 